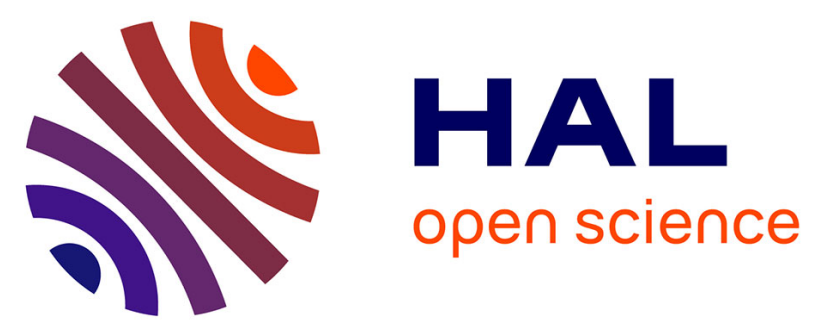

\title{
A Proposal for a Classification for Recurrent Endometrial Cancer: Analysis of a French Multicenter Database From the FRANCOGYN Study Group
}

\author{
Alexandre Bricou, Sofiane Bendifallah, Mathilde Daix-Moreux, Lobna \\ Ouldamer, Vincent Lavoué, Amélie Benbara, Cyrille Huchon, Geoffroy \\ Canlorbe, Emilie Raimond, Charles Coutant, et al.
}

\section{To cite this version:}

Alexandre Bricou, Sofiane Bendifallah, Mathilde Daix-Moreux, Lobna Ouldamer, Vincent Lavoué, et al.. A Proposal for a Classification for Recurrent Endometrial Cancer: Analysis of a French Multicenter Database From the FRANCOGYN Study Group. International Journal of Gynecological Cancer, 2018, 28 (7), pp.1278-1284. 10.1097/IGC.0000000000001296 . hal-02111165

\section{HAL Id: hal-02111165 \\ https://hal-amu.archives-ouvertes.fr/hal-02111165}

Submitted on 25 Apr 2019

HAL is a multi-disciplinary open access archive for the deposit and dissemination of scientific research documents, whether they are published or not. The documents may come from teaching and research institutions in France or abroad, or from public or private research centers.
L'archive ouverte pluridisciplinaire HAL, est destinée au dépôt et à la diffusion de documents scientifiques de niveau recherche, publiés ou non, émanant des établissements d'enseignement et de recherche français ou étrangers, des laboratoires publics ou privés. 


\title{
A Proposal for a Classification for Recurrent Endometrial Cancer
}

\author{
Analysis of a French Multicenter Database From the \\ FRANCOGYN Study Group
}

\author{
Alexandre Bricou, MD, * Sofiane Bendifallah, MD, PhD, † Mathilde Daix-Moreux, MD,* \\ Lobna Ouldamer, MD, PhD, $\neq$ Vincent Lavoue, MD, PhD, $\S$ Amélie Benbara, MD,* \\ Cyrille Huchon, MD, PhD,// Geoffroy Canlorbe, MD, PhD, $\dagger$ Emilie Raimond, MD, $\mathbb{I}$ \\ Charles Coutant, MD, PhD,\# Olivier Graesslin, MD, PhD, I Pierre Collinet, MD, PhD, ** \\ Xavier Carcopino, $M D, P h D, \dagger \dagger$ Cyril Touboul, $M D, P h D,+\$ \oint \oint$ Emile Daraï, MD, PhD, $\dagger$ \\ Lionel Carbillon, MD, PhD, * and Marcos Ballester, MD, PhD, $\dagger$ \\ for the Groupe de Recherche FRANCOGYN
}

\begin{abstract}
Objective: Endometrial cancer (EC) recurrences are relatively common with no standardized way of describing them. We propose a new classification for them called locoregional, nodal, metastasis, carcinomatosis recurrences (rLMNC).

Patients and Methods: The data of 1230 women with EC who were initially treated by primary surgery were included in this French multicenter retrospective study. Recurrences were classified based on dissemination pathways: (1) locoregional recurrence (rL); (2) nodal recurrence (rN) for lymphatic pathway; (3) distant organ recurrence (rM) for hematogenous pathway; and (4) carcinomatosis recurrence ( $\mathrm{rC}$ ) for peritoneal pathway. These pathways were further divided into subgroups. We compared recurrence free survival and overall survival (OS) between the 4 groups ( $\mathrm{rL} / \mathrm{rN} / \mathrm{rM} / \mathrm{rC}$ ).

Results: The median follow-up was 35.6 months (range, 1.70-167.60). One hundred ninety-eight women (18.2\%) experienced a recurrence: 150 (75.8\%) experienced a singlepathway recurrence and $48(24.2 \%)$ a multiple-pathway recurrence. The 5 -year OS was $34.1 \%$ (95\% confidence interval [CI], 27.02\%-43.1\%), and the median time to first recurrence was 18.9 months (range, $0-152$ months). The median survival after recurrence was 14.8 months $(95 \%$ CI, $11.7-18.8)$. Among women with single pathway of recurrence, a difference in 5-year
\end{abstract}

\footnotetext{
*Department of Obstetrics and Gynecology, Jean-Verdier University Hospital, Assistance Publique des Hôpitaux de Paris, University Paris 13; †Department of Gynaecology and Obstetrics, Tenon University Hospital, Assistance Publique des Hôpitaux de Paris, University Pierre and Marie Curie; \$Department of Obstetrics and Gynaecology, Centre Hospitalier Régional Universitaire de Tours, Hôpital Bretonneau, Tours; §CRLCC Eugène-Marquis, Service de Gynécologie, CHU de Rennes, Université de Rennes 1; |Department of Gynaecology and

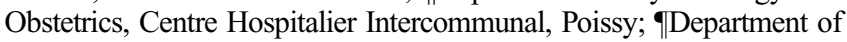
Obstetrics and Gynaecology, Institute Alix de Champagne University Hospital, Reims; \#Centre de Lutte Contre le Cancer Georges François Leclerc, Dijon; **Department of Obstetrics and Gynaecology, Centre
}

Hospitalier Régional Universitaire, Lille; ††Department of Obstetrics and Gynecology, Hôpital Nord, Marseille; +tDepartment of Obstetrics and Gynecology, Centre Hospitalier Intercommunal, Créteil; and $\S \S$ Faculté de Médecine de Créteil, UPEC-Paris XII, Paris, France. Address correspondence and reprint requests to Bricou Alexandre, MD, Department of Obstetrics and Gynecology, Jean-Verdier University Hospital, Assistance Publique des Hôpitaux de Paris, University Paris 13, Avenue du 14 Juillet, 93143 Bondy Cedex, France. E-mail: alexandre.bricou@aphp.fr.

The authors declare no conflict of interest.

Clinical trial identification number: CEROG 2014-GYN-020.

URL of the registration site: http://www.cngof.fr/accueil-cngof/ le-cerog. 
OS $(P<0.001)$ and survival after recurrence $(P<0.01)$ was found between the $4 \mathrm{rLNMC}$ groups. The carcinomatosis group had the worst prognosis compared with other single recurrence pathways. Women with multiple recurrences had poorer 5-year OS $(P<0.001)$ and survival after recurrence $(P<0.01)$ than those with single metastasis recurrence, other than women with peritoneal carcinomatosis.

Conclusions: This easy-to-use and intuitive classification may be helpful to define EC recurrence risk groups and develop guidelines for the management of recurrence. Its prognosis value could also be a tool to select homogenous populations for further trials.

Key Words: Endometrial cancer, Recurrences, Relapses, Classification

E ndometrial cancer $(\mathrm{EC})$ is the most common gynecologic Eancer worldwide with 320,000 new cases each year. ${ }^{1}$ Around $75 \%$ of ECs are diagnosed at an early stage with a 5 -year overall survival (OS) reaching $80 \%{ }^{2}$ However, EC is a heterogeneous disease with some subsets of women displaying an increased risk of recurrence. Indeed, it causes almost 76,000 deaths per year. ${ }^{1}$ The most usual identified risk factors for recurrence are the International Federation of Gynecology and Obstetrics (FIGO) stage, depth of myometrial invasion, histological type and grade, lymphovascular space invasion, and tumor size. $^{3}$ In this specific setting, the European Society for Medical Oncology (ESMO)/European Society of Gynaecological Oncology/European Society for Radiotherapy and Oncology group recently classified EC women into 4 subgroups according to the risk of recurrence (low risk, intermediate risk, highintermediate risk, and high risk) to better adapt surgical staging and indications for adjuvant therapies. ${ }^{4}$

Although overall prognosis of women with surgically treated EC is good, Bendifallah et $\mathrm{al}^{5}$ recently reported that at least $20 \%$ of them will experience a recurrence, impacting OS. Moreover, it is now well recognized that more than $70 \%$ of recurrences occur within the first 2 to 3 years after initial treatment. ${ }^{6-8}$ Hence, recurrence events and their treatment are a crucial aspect in the management of women with EC. Most studies to date focus on predictive factors of recurrence, recurrence patterns, and specific treatments. ${ }^{9-11}$ In this setting, most authors concur that locoregional recurrences are to be differentiated from distant recurrences.

However, there is currently no consensual standardized description of EC recurrence and definitions vary widely from one study to another. ${ }^{12-16}$ This lack of homogeneity limits comparison of study results by rendering their interpretation somewhat blurred.

Hence, the purpose of this study was to describe the anatomical locations of EC recurrence with a view to developing an easy-to-use classification based on the 4 dissemination pathways (locoregional, lymphatic, blood, and peritoneal) to better assess prognosis.

\section{PATIENTS AND METHODS}

\section{Study Population}

From January 2001 to December 2013, data of women with histologically proven EC who received primary surgical treatment were abstracted from EC databases of 9 institutions in France (Tours University Hospital, Tenon University Hospital, Dijon Cancer Centre, Rennes University Hospital, Lille University Hospital, Reims University Hospital, Creteil University Hospital, Poissy University Hospital, and Jean Verdier University Hospital) and from the Senti-Endo trial. ${ }^{17}$ The research protocol was approved by the Institutional Review Board of the Collège National des Gynécologues et Obstétriciens Français (CEROG 2014-GYN-020).

All enrolled women underwent preoperative imaging examinations (abdominopelvic magnetic resonance imaging and/or computed tomography scan). The following clinical, surgical, pathological, and adjuvant therapy data were collected: women's age, body mass index (BMI; calculated as weight in kilograms divided by the square of height in meters), surgical procedure, nodal staging, final pathological analysis (histological type and grade, depth of myometrial invasion, and lymphovascular space invasion status), and type of adjuvant therapy. Histological type 1 included endometrioid tumors (grades 1 to 3 ), and histological type 2 included serous or clearcell carcinomas and carcinosarcomas. All women were classified according to the FIGO 2009 classification ${ }^{18}$ after final pathological analysis and were classified into recurrence risk groups as defined by the ESMO/European Society of Gynaecological Oncology/European Society for Radiotherapy and Oncology guidelines. ${ }^{4}$

\section{Treatment and Follow-up}

All the women underwent primary surgical treatment including at least total hysterectomy with bilateral salpingo-oophorectomy, with or without nodal staging (sentinel lymph nodes \pm pelvic \pm para-aortic lymphadenectomy), as well as omentectomy. Adjuvant therapy and clinical follow-up were decided by a 
multidisciplinary committee and based on the French guidelines. Adjuvant therapy could include vaginal brachytherapy and/or external beam radiotherapy (EBRT) and/or chemotherapy (CT). Clinical follow-up consisted of physical examinations and the use of imaging techniques according to the findings. Follow-up visits were conducted every 3 months for the first 2 years, every 6 months for the following 3 years, and once a year thereafter.

\section{Recurrence Events and Classification}

Recurrent disease was assessed by physical examination and imaging techniques (computed tomography, magnetic resonance imaging, ultrasonography, bone scintigraphy, positron emission tomography with the fluorodesoxyglucose $(18 \mathrm{~F})$ as well as histological findings when feasible. We applied a new classification, called the rLNMC classification, based on the pathway of dissemination: locoregional recurrence $(\mathrm{rL})$, nodal recurrence $(\mathrm{rN})$, distant organ recurrence $(\mathrm{rM})$, and peritoneal recurrence with carcinomatosis ( $\mathrm{rC}$ ) (Table 1). Locoregional pathway recurrence was divided into 2 groups as follows: recurrence in the vaginal vault only (rL1) and rectal and/or bladder involvement (rL2). We defined a locoregional recurrence that could not be assessed (rLx) and no evidence of

\section{TABLE 1. rLMNC classification for EC recurrence}

\begin{tabular}{ll}
\hline rLocoregional & \\
\hline $\mathrm{rLx}$ & A recurrence tumor that cannot be assessed \\
$\mathrm{rL0}$ & No evidence of the recurrent tumor site \\
$\mathrm{rL1}$ & Recurrence tumor in the vaginal vault only \\
$\mathrm{rL2}$ & $\begin{array}{c}\text { Centropelvic recurrence tumor with or } \\
\text { without rectal and/or bladder } \\
\end{array}$ \\
& involvement. \\
\hline
\end{tabular}

rNode

\begin{tabular}{ll}
\hline $\mathrm{rNx}$ & $\begin{array}{c}\text { The lymph node recurrence cannot } \\
\text { be assessed }\end{array}$ \\
$\mathrm{rN} 0$ & $\begin{array}{c}\text { No lymph node recurrence } \\
\text { Lymphnode recurrence in pelvic, inguinal, } \\
\text { and/or infradiaphragmatic para-aortic area }\end{array}$ \\
$\mathrm{rN2}$ & $\begin{array}{c}\text { Mediastinal or other distance lymph } \\
\text { node recurrence }\end{array}$ \\
\hline
\end{tabular}

rMetastasis

\begin{tabular}{ll}
\hline rMx & Distant organ recurrence cannot be assessed \\
rM0 & No distant organ recurrence \\
rM1 & Distant recurrence in one organ \\
rM2 & Distant recurrence in two or more organs. \\
\hline
\end{tabular}

rCarcinomatosis

$\mathrm{rCx} \quad$ Carcinomatosis recurrence cannot be assessed

$\mathrm{rC0} \quad$ No carcinomatosis recurrence

rC1 Presence of carcinomatosis recurrence locoregional recurrence as rL0. Nodal recurrences were separated into 2 groups: for lymph node recurrence in the pelvic, inguinal, and/or infradiaphragmatic para-aortic area (rN1) and for mediastinal or other distance nodal recurrences (rN2). We defined a node recurrence that could not be assessed as $\mathrm{rNx}$ and no evidence of the recurrent node site as rNO. Hematogenous recurrences were also divided into 2 groups: single metastasis (rM1) and multiple metastasis (rM2). We defined a metastatic recurrence that could not be assessed as (rMx) and no evidence of the metastatic as (rM0). The peritoneal recurrence represented a single group for pelvic and/or abdominal carcinomatosis $(\mathrm{rC} 1)$. We defined a peritoneal recurrence that could not be assessed as $\mathrm{rCx}$ and no evidence of carcinomatosis as (rC0). Single recurrence was defined as recurrence with only one pathway of dissemination. Multiple recurrences were defined as recurrences with more than one pathway of dissemination, Supplemental Digital Content 1, http://links.lww.com/IGC/A754;Supplemental Digital Content 2, http://links.lww.com/IGC/A755.

\section{Statistical Analysis}

Recurrence free survival was defined as the length of time from the date of primary surgery to any EC recurrence and was censored at the date of the last follow-up or date of death without recurrence. Overall survival was defined as time from primary surgery to death as a result of any cause and survival after recurrence was defined as time from recurrence diagnostic to death. Kaplan-Meier estimates were used to estimate the eventtime distributions, and log-rank test was used to compare the differences in terms of OS between the 4 groups $(\mathrm{rL} / \mathrm{rN} / \mathrm{rM} / \mathrm{rC})$. Values of $P<0.05$ were considered to denote significant differences. Data were managed with an Excel database (Microsoft, Redmond, Wash) and analyzed using R 2.15 software, available online with caTools, rms, presence/absence, and verification libraries (https://www.r-project.org/).

\section{RESULTS}

\section{Characteristics of the Population}

During the study period, 1230 women with EC who were initially treated by primary surgery were included in this multicenter retrospective study. Two hundred twenty-four women (18.2\%) experienced a recurrence. Among them, 26 were excluded owing to incomplete data leaving 198 women for final analysis. The median age of the women with recurrence was 68.5 years (range, $32-88$ years), and their BMI was $29.09 \mathrm{~kg} / \mathrm{m}^{2}$ (range, $14-50.4 \mathrm{~kg} / \mathrm{m}^{2}$ ). The median follow-up was 35.6 months (range, 1.70-167.60 months) after primary diagnosis and 10.2 months (range, 1-109 months) after recurrence. The median recurrence free survival was 18.9 months (range, $0-152$ months). The 5-year OS was 34.1\% (95\% CI, 27.02\%-43.1\%). The median survival after recurrence was 14.8 months ( $95 \%$ CI, $11.7-18.8)$. One hundred nineteen women $(60 \%)$ died, and $65(55 \%)$ of these deaths were due to EC recurrence. For the remaining 54 women, the cause of the death was not recorded. The characteristics of the women with recurrence are reported in Table 2. 
TABLE 2. Characteristics of women who experienced a recurrence

\begin{tabular}{lc}
\hline Characteristics & Population, $\mathbf{n}=\mathbf{1 9 8}$ \\
\hline Age, median (range), y & $68.49(32-88)$ \\
BMI, median (range), $\mathrm{kg} / \mathrm{m}^{2}$ & $29.09(14-50.4)$ \\
Follow-up, median (range), mo & $35.6(1.70-167.60)$ \\
Histological type & $\mathrm{n}(\%)$ \\
Type 1 & $115(58)$ \\
Type 2 & $83(42)$ \\
ESMO classification & $\mathrm{n}(\%)$ \\
Low risk & $18(9.1)$ \\
Intermediate risk & $9(4.5)$ \\
Intermediate-high risk & $18(9.1)$ \\
High risk & $151(76.3)$ \\
NA & $2(1)$ \\
FIGO stage at definitive histology & $\mathrm{n}(\%)$ \\
I & $86(43.4)$ \\
II & $26(13.1)$ \\
III & $73(36.9)$ \\
IV & $13(6.6)(13)$ \\
Adjuvant therapies before recurrence & $\mathrm{n}(\%)$ \\
No adjuvant therapy & $30(15.2)$ \\
Vaginal brachytherapy alone & $13(6.6)$ \\
EBRT + brachytherapy & $64(32.3)$ \\
CT + EBRT & $21(10.6)$ \\
CT alone & $28(14.1)$ \\
EBRT + brachytherapy + CT & $27(13.6)$ \\
EBRT alone & $14(7.1)$ \\
Brachytherapy + CT & $1(0.5)$ \\
\hline NA, not available. & \\
\hline &
\end{tabular}

\section{Patterns of Recurrence According to the rLNMC Classification}

Among the 198 women who experienced recurrence: $52(21.1 \%)$ experienced a locoregional recurrence (vaginal vault [ $\mathrm{rL} 1]$ in 22 cases [8.9\%], centropelvic recurrence [ $\mathrm{rL} 2]$ in 30 cases [12.2\%]); 50 (20.3\%) experienced a nodal recurrence (pelvic, inguinal, and/or para-aortic node recurrence [rN1] in 41 cases [16.7\%] and mediastinal or distant nodes [rN2] in 9 cases [3.8\%]); 95 (38.6\%) experienced a metastatic pathway recurrence (rM1) in 49 cases $(19.9 \%)$ and rM2 in 46 cases $(18.7 \%)$; and $49(19.9 \%)$ experienced a peritoneal carcinomatosis recurrence $(\mathrm{rC} 1)$.

Among the women who experienced a single recurrence $(\mathrm{n}=150,75.8 \%): 30(20 \%)$ experienced a tumor site recurrence (vaginal recurrence [rL1N0M0C0] in 14 cases [46.7\%], centropelvic recurrence [rL2N0M0C0] in 16 cases [53.3\%]); 26 (17.3\%) experienced a nodal recurrence (pelvic, para-aortic, or inguinal areas [rL0N1M0C0] in 18 cases [69.2\%] and mediastinal or distance nodes [rL0N2M0C0] in
8 cases [30.8\%]); and $65(43.3 \%)$ experienced a metastatic recurrence (only 1 localization [rL0N0M1C0] in 32 cases [49.2\%] [15 lung, 8 bone, 6 brain, 2 liver, 1 unspecified], more than 1 [rL0N0M2C0] in 33 cases [50.8\%], and peritoneal carcinomatosis recurrence (rL0NOM0C1) in 29 cases [19.4\%]).

Among the women who experienced a multiple recurrence $(n=48[24.2 \%]): 22$ (45.8\%) experienced a locoregional recurrence (vaginal vault [rL1] in 8 cases [36.4\%], centropelvic [rL2] in 14 cases [63.6\%]); 24 (50\%) experienced a nodal recurrence (involving pelvic, para-aortic, or inguinal area nodes [rN1] in 23 cases [95.8\%] and mediastinal area or distance nodes [rN2] in 1 case [4.2\%]); and $30(62.5 \%)$ experienced a metastatic recurrence ( 1 metastasis [8 lung, 7 liver, 1 unspecified, 1 bone] [rM1] in 17 cases [56.7\%], more than 1 metastatic recurrence [rM2] in 13 cases [43.3\%] [Table 3], and peritoneal carcinomatosis [rC1] in 20 cases [41.7\%]).

\section{Survival Outcomes}

The 5-year OS was $34.1 \%$ (95\% CI, 27,02-43,1\%) (Fig. 1A). The median survival after recurrence was 14.8 months (95\% CI, 11.7-18.8) (Fig. 1B). Among women with single pathway of recurrence, a difference in 5-year OS (Fig. 2A) and survival after recurrence (Fig. 2B) was found between the $4 \mathrm{rLNMC}$ groups. The carcinomatosis group (rL0N0M0C1) had the worst prognosis compared with other single recurrence pathways. When considering women with multiple recurrences, a difference in 5-year OS (Fig. 3A) and survival after recurrence (Fig. 3B) was found between the rLNMC groups.

\section{DISCUSSION}

Our study confirms that the patterns of recurrence in EC are multiple. Moreover, it shows that recurrence sites can be isolated or associated with each other: we identified 246 different recurrences among the 198 women who experienced recurrence with $75.8 \%$ experiencing single recurrence and $(24.2 \%)$ multiple recurrences. These results are in agreement with those of Sohaib et al ${ }^{12}$ who report $48 \%$ of multiple-site recurrences. This is why the rLNMC classification we propose here includes the different pathways of spread: locoregional

TABLE 3. Women with single or multipathways of recurrence with rLNMC classification

\begin{tabular}{lc}
\hline Single recurrence on locoregional site $(\mathrm{rL})$ & $30(15.2 \%)$ \\
rL1N0M0C0 & 14 \\
rL2N0M0C0 & 16 \\
Single-nodes recurrence $(\mathrm{rN})$ & $26(13.2 \%)$ \\
rL0N1M0C0 & 18 \\
rL0N2M0C0 & 8 \\
Single-metastasis recurrence (rM) & $65(32.8 \%)$ \\
rL0N0M1C0 & 32 \\
rL0N0M2C0 & 33 \\
Single-carcinomatosis recurrence (Rc) & $29(14.6 \%)$ \\
rL0N0M0C1 & 29 \\
Multiple-pathways recurrence (rMpath) & $48(24.2 \%)$ \\
\hline
\end{tabular}


a
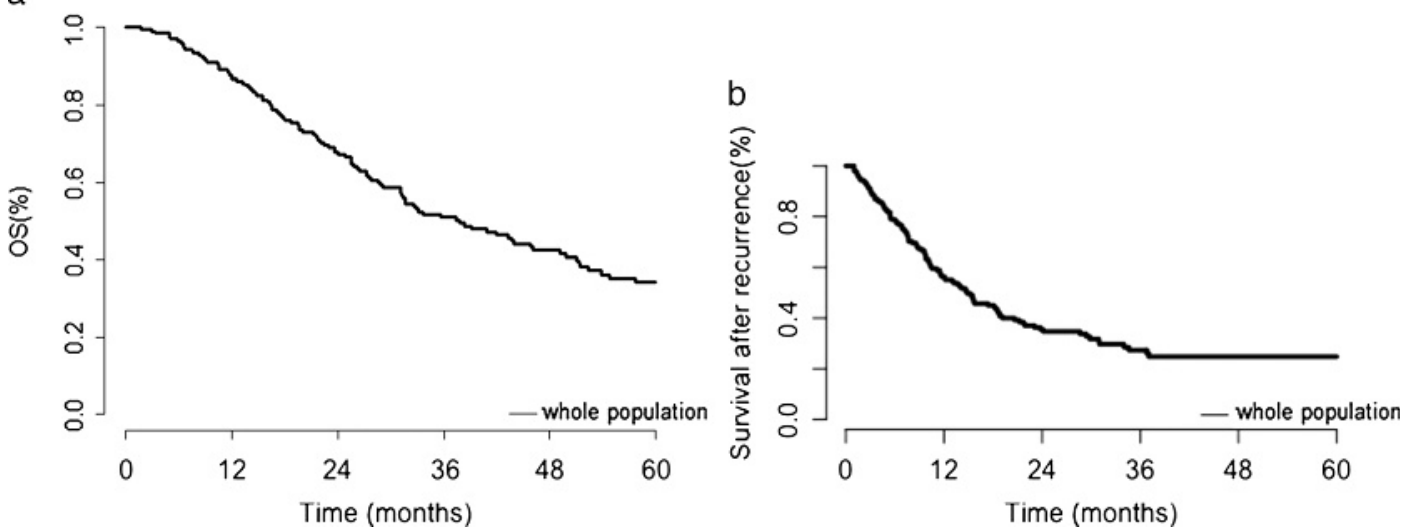

FIGURE 1. A, Five-year cumulative survival rates for the whole population. B, Cumulative survival rates after recurrence for the whole population.

extension (contiguous extension), lymphatic dissemination (nodal recurrences), hematogenous dissemination (metastatic recurrences), and peritoneal seeding (carcinomatosis). It is based on clinical and/or imaging data found during the follow-up period with or without histological confirmation and describes localization, type, and focality of recurrences. Moreover, localization of recurrence is not the only prognostic factor; prognosis may also depend on the type of recurrence treatment, which is conditioned by (1) initial treatment (radiotherapy), (2) the interval between initial diagnosis and recurrence, and (3) the initial histology and tumor grade. ${ }^{13}$ Hence, our classification could be completed by the type of early adjuvant treatment used. Consequently, the prefixes $\mathrm{Ra}$ for radiotherapy, $\mathrm{Ch}$ for $\mathrm{CT}$, and $\mathrm{Ht}$ for hormonotherapy could also be recorded in the classification.

In the classification we present here, locoregional recurrence $(\mathrm{rL})$ concerns recurrences localized in the locoregional area and related to contiguous dissemination (from the initial EC tumor site). In this specific setting, we decided to exclude parenchimatous recurrences from this group and include them in the hematogenous pathway (metastasis) group, even if such recurrences can involve abdominal or pelvic organs. Our division into 2 different subgroups ( $\mathrm{rL} 1, \mathrm{rL} 2)$ is supported by its impact on OS: a trend for poorer prognosis was found for centropelvic recurrences (rL2, N0, M0, C0) compared with vaginal vault recurrences ( $\mathrm{rL1}, \mathrm{N} 0, \mathrm{M} 0, \mathrm{C} 0)$. Therapeutic options are an additional parameter supporting this distribution. Indeed, surgical treatment can be more difficult and sometimes not possible for centropelvic recurrences as opposed to isolated vaginal vault recurrence, which can often be salvaged with surgical excision and/or radiotherapy. In this setting, Ackerman et al ${ }^{19}$ reported a local control rate of up to $79 \%$ for isolated vaginal vault recurrences. Moreover, several studies report that survival is better in women with recurrence confined within the vaginal vault rather than in the true pelvis ${ }^{13,20-22}$ or other sites. ${ }^{23}$ However, these results must be interpreted with caution because the prognosis seems to be the poorest for women who initially received external beam radiotherapy. ${ }^{13}$ Concerning centropelvic recurrences (rL2), the extent of recurrence appears to be an important prognostic factor for local control and survival. ${ }^{24}$ In a series of 209 women with recurrence, Sartori et $\mathrm{al}^{23}$ reported that the 5-year OS for vaginal, pelvic, and distant recurrence was $68 \%, 29 \%$, and $8 \%$, respectively.

Concerning the lymphatic pathway, nodal recurrences represented $20.3 \%$ of the total recurrence sites in our study and were isolated in $13.1 \%$ of cases. These findings are in contradiction with those of Sohaib et $\mathrm{al}^{12}$ who found that nodal recurrences were the most frequent recurrence site $(47 \%)$. a

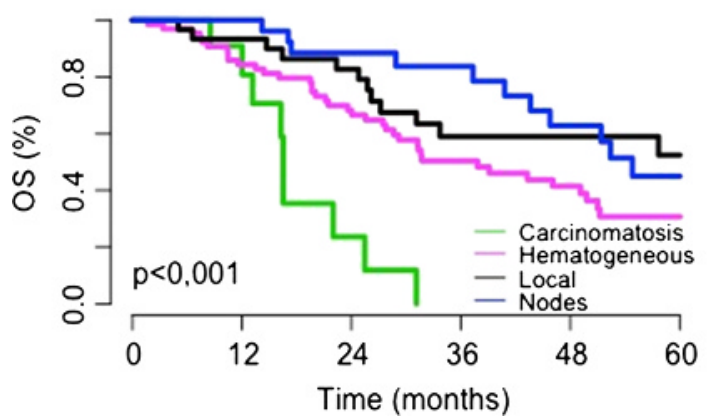

b

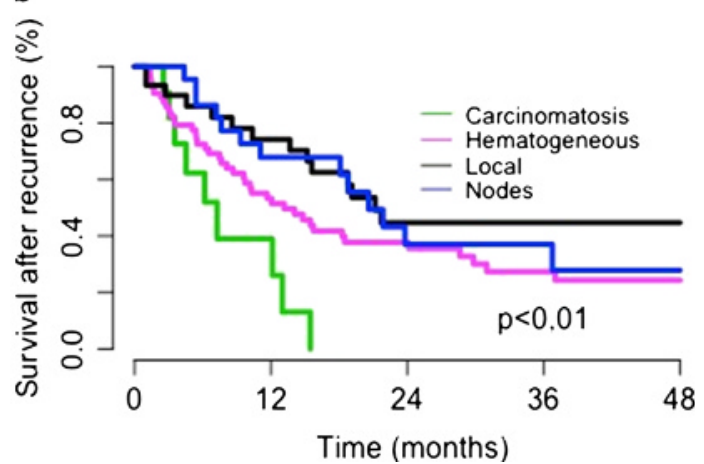

FIGURE 2. A, Five-year cumulative survival rates for the different single recurrences. B, Cumulative survival rates after recurrence for different single recurrences. 
a

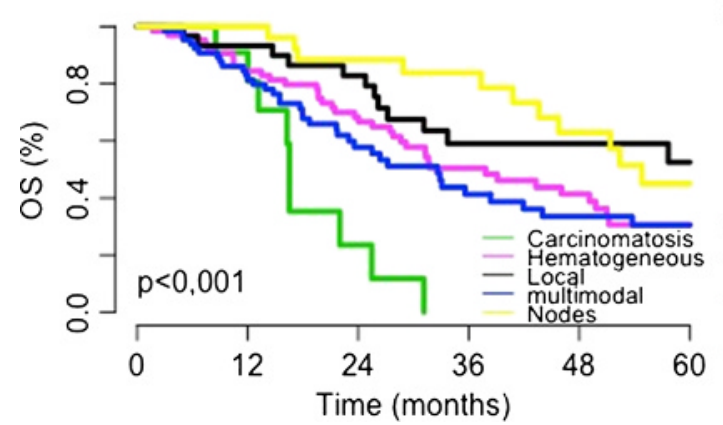

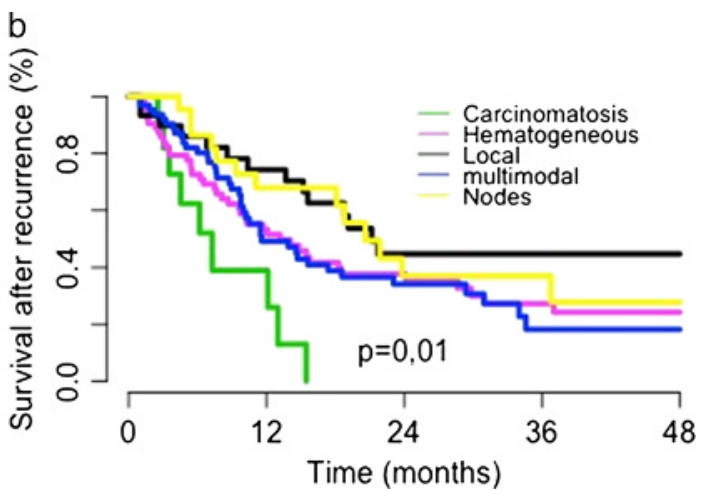

FIGURE 3. A, Five-year cumulative survival rates for single and multiple recurrences. $B$, Cumulative survival rates after recurrences for single and multiple recurrences.

However, their study included 86 patients among whom only 40 (46.5\%) had undergone nodal dissection during primary surgery. ${ }^{12}$ We distinguish between 2 types nodal recurrence in our classification: $\mathrm{rN1}$ for all pelvic, inguinal, and/or infradiaphragmatic para-aortic area node recurrence and $\mathrm{rN} 2$ for mediastinal or other distant lymph node recurrence. This distinction is based on the possibility of surgical removal for the $\mathrm{rN} 1$ group compared with the $\mathrm{rN} 2$ group. However, we were not able to distinguish pelvic node involvement from infradiaphragmatic para-aortic or inguinal nodal involvement in the database. Moreover, we did not find any difference in survival between the rN1 and the rN2 groups, when isolated. This can be explained by the fact that isolated $\mathrm{rN} 2$ recurrences are rare and that nodal recurrences are often associated with other recurrence sites. However, a few studies in the literature have attempted to distinguish between type of nodal involvement and what we consider to be the rN2 group is often classified as distant recurrence sites. ${ }^{5,21,22,25}$

In our study, metastases represented the first pathway of recurrence and was found in 95 women (38.6\%) with a similar distribution between isolated and multiple metastases. This rate is in complete agreement with that reported by Sohaid et $\mathrm{al}^{12}$ who found that metastases occurred in $36 \%$ of recurrences and represented a major predictor factor for poor survival. In the current classification, we decided to classify metastatic recurrences as single (rM1) or multiple (rM2). We believe that single metastases could be, in selected cases, eligible for local control (surgery or interventional imaging) with a view to increasing the symptom-free interval, which is less feasible for multiple-site metastases. In contrast, we decided not to distinguish between the different anatomical sites because this would detract from the clarity and ease-of-use of the classification.

In our study, the presence of peritoneal carcinomatosis was linked to a significant decrease in 5-years OS and survival after recurrence. That is why we decided to create a single group because it may represent a specific pathway of dissemination. In the current literature, the real impact of peritoneal carcinomatosis on survival remains unclear because it is often associated with other recurrence sites such as distant metastases, extravaginal recurrences, or abdominal recurrences. ${ }^{22,26-28}$ Indeed, Ouldamer et $\mathrm{al}^{29}$ recently reported a nomogram to predict poor prognosis recurrences (ie, peritoneal carcinomatosis and distant metastases) with a 3 -year OS of $33.1 \%$ for the peritoneal carcinomatosis group. Finally, the treatment options may differ depending on whether the peritoneal carcinomatosis is isolated or associated to another pathway of dissemination. In this setting, in case of isolated carcinomatosis, the place of cytoreductive surgery with hyperthermic intraperitonal CT is a matter of major interest.

The strengths of our study lie in its multicenter nature and the large number of women with EC recurrence included. However, we cannot exclude an inherent bias linked to its retrospective nature. Indeed, among the 224 women who experienced a recurrence, $26(11.6 \%)$ were excluded owing to incomplete data. Moreover, our database did not allow to differentiate pelvic nodes involvement, infradiaphragmatic paraaortic area nodes involvement, and inguinal nodes involvement. Finally, during the data collection period, modifications occurred in surgical techniques such as lymph node staging and indications for adjuvant therapies with potential impact on prognosis. However, all women were managed in regional referral centers with a systematic multidisciplinary committee approval in accordance with French/Europeans guidelines. ${ }^{30}$

In conclusion, EC recurrences represent a heterogeneous group of women with various histological characteristics, initial treatments, and anatomical sites of recurrence. Such heterogeneity has been widely described, but, to our knowledge, this is the first study to propose a classification including standardized definitions of recurrence based on the different pathways of dissemination. A consensual classification would not only make it easier to better define risk groups and develop guidelines for the surgical or medical management of EC recurrence but also help select homogenous populations for further trials. This first classification for EC recurrence should, naturally, be further developed and updated.

\section{REFERENCES}

1. Galaal K, Al Moundhri M, Bryant A, et al. Adjuvant chemotherapy for advanced endometrial cancer. Cochrane Database Syst Rev. 2014;5:CD010681.

2. Amant F, Moerman P, Neven P, et al. Endometrial cancer. Lancet Lond Engl. 2005;366:491-505.

3. Morrow CP, Bundy BN, Kurman RJ, et al. Relationship between surgical-pathological risk factors and outcome in clinical stage I 
and II carcinoma of the endometrium: a Gynecologic Oncology Group study. Gynecol Oncol. 1991;40:55-65.

4. Colombo N, Creutzberg C, Amant F, et al. ESMO-ESGO-ESTRO consensus conference on endometrial cancer: diagnosis, treatment and follow-up. Radiother Oncol. 2015;117:559-581.

5. Bendifallah S, Ouldamer L, Lavoue V, et al. Patterns of recurrence and outcomes in surgically treated women with endometrial cancer according to ESMO-ESGO-ESTRO Consensus Conference risk groups: results from the FRANCOGYN study Group. Gynecol Oncol. 2017;144: 107-112.

6. Fung-Kee-Fung M, Dodge J, Elit L, et al. Follow-up after primary therapy for endometrial cancer: a systematic review. Gynecol Oncol. 2006;101:520-529.

7. Ben Arie A, Lavie O, Gdalevich M, et al. Temporal pattern of recurrence of stage I endometrial cancer in relation to histological risk factors. Eur J Surg Oncol. 2012;38:166-169.

8. Kilgore JE, Jackson AL, Ko EM, et al. Recurrence-free and 5 -year survival following robotic-assisted surgical staging for endometrial carcinoma. Gynecol Oncol. 2013;129:49-53.

9. Rauh-Hain JA, Del Carmen MG. Treatment for advanced and recurrent endometrial carcinoma: combined modalities. Oncologist. 2010;15:852-861.

10. Del Carmen MG, Boruta DM, Schorge JO. Recurrent endometrial cancer. Clin Obstet Gynecol. 2011;54:266-277.

11. Morice P, Leary A, Creutzberg C, et al. Endometrial cancer. Lancet. 2016;387:1094-1108.

12. Sohaib SA, Houghton SL, Meroni R, et al. Recurrent endometrial cancer: patterns of recurrent disease and assessment of prognosis. Clin Radiol. 2007;62:28-34; discussion 35-36.

13. Creutzberg CL, van Putten WL, Koper PC, et al. Survival after relapse in patients with endometrial cancer: results from a randomized trial. Gynecol Oncol. 2003;89:201-209.

14. Barlin JN, Wysham WZ, Ferda AM, et al. Location of disease in patients who die from endometrial cancer: a study of 414 patients from a single institution. Int J Gynecol Cancer. 2012;22:1527-1531.

15. Simpkins F, Papadia A, Kunos C, et al. Patterns of recurrence in stage I endometrioid endometrial adenocarcinoma with lymphovascular space invasion. Int J Gynecol Cancer. 2013;23:98-104.

16. Wang J, Jia N, Li Q, et al. Analysis of recurrence and survival rates in grade 3 endometrioid endometrial carcinoma. Oncol Lett. 2016;12:2860-2867.

17. Ballester M, Dubernard G, Lécuru F, et al. Detection rate and diagnostic accuracy of sentinel-node biopsy in early stage endometrial cancer: a prospective multicentre study (SENTI-ENDO). Lancet Oncol. 2011;12:469-476.
18. Pecorelli S. Revised FIGO staging for carcinoma of the vulva, cervix, and endometrium. Int J Gynaecol Obstet. 2009;105: 103-104.

19. Ackerman I, Malone S, Thomas G, et al. Endometrial carcinoma - relative effectiveness of adjuvant irradiation vs therapy reserved for relapse. Gynecol Oncol. 1996;60:177-183.

20. Jeppesen MM, Jensen PT, Gilså Hansen D, et al. The nature of early-stage endometrial cancer recurrence- a national cohort study. Eur J Cancer. 2016;69:51-60.

21. Sartori E, Pasinetti B, Carrara L, et al. Pattern of failure and value of follow-up procedures in endometrial and cervical cancer patients. Gynecol Oncol. 2007:107(1 Suppl 1):S241-S247.

22. Esselen KM, Boruta DM, del Carmen M, et al. Defining prognostic variables in recurrent endometrioid endometrial cancer: a 15-year single-institution review. Int J Gynecol Cancer. 2011;21:1078-1083.

23. Sartori E, Laface B, Gadducci A, et al. Factors influencing survival in endometrial cancer relapsing patients: a Cooperation Task Force (CTF) study. Int J Gynecol Cancer. 2003;13: 458-465.

24. Curran WJ Jr, Whittington R, Peters AJ, et al. Vaginal recurrences of endometrial carcinoma: the prognostic value of staging by a primary vaginal carcinoma system. Int $J$ Radiat Oncol Biol Phys. 1988;15:803-808.

25. Tejerizo-García A, Álvarez-Conejo C, Muñoz-Hernando L, et al. Tumor recurrence and tumor-related mortality in endometrial cancer: analysis in 276 patients. Indian J Cancer. 2015;52:682-684.

26. Moschiano EJ, Barbuto DA, Walsh C, et al. Risk factors for recurrence and prognosis of low-grade endometrial adenocarcinoma; vaginal versus other sites. Int J Gynecol Pathol. 201433:268-273.

27. Dunn EF, Geye H, Platta CS, et al. Predictive factors of recurrence following adjuvant vaginal cuff brachytherapy alone for stage I endometrial cancer. Gynecol Oncol. 2014;133: 494-498.

28. Odagiri T, Watari H, Hosaka M, et al. Multivariate survival analysis of the patients with recurrent endometrial cancer. J Gynecol Oncol. 2011;22:3-8.

29. Ouldamer L, Bendifallah S, Body G, et al. Predicting poor prognosis recurrence in women with endometrial cancer: a nomogram developed by the FRANCOGYN study group. Br J Cancer. 2016;115:1296-1303.

30. Querleu D, Planchamp F, Narducci F, et al. Clinical practice guidelines for the management of patients with endometrial cancer in France: recommendations of the Institut National du Cancer and the Société Française d'Oncologie Gynécologique. Int J Gynecol Cancer. 2011;21:945-950. 